\title{
New directions in the Minimal Model Program
}

\author{
Paolo Cascini ${ }^{1}$
}

Received: 15 April 2020 / Accepted: 17 July 2020 / Published online: 12 September 2020

(c) The Author(s) 2020

\begin{abstract}
We survey some recents developments in the Minimal Model Program. After an elementary introduction to the program, we focus on its generalisations to the category of foliated varieties and the category of varieties defined over any algebraically closed field of positive characteristic.
\end{abstract}

\section{Contents}

1 Introduction . . . . . . . . . . . . . . . . . . . . . . . . 179

2 Classical Minimal Model Program _. . . . . . . . . . . . . . . . . . . . . . . . 181

2.1 K-stable varieties . . . . . . . . . . . . . . . . . . . . . . . . . . . . . . . . . 182

2.2 Boundedness and moduli spaces . . . . . . . . . . . . . . . . . . . . . . . 183

3 Minimal Model Program for foliations . . . . . . . . . . . . . . . . . . . . . . . . 185

4 Minimal Model Program in positive characteristic . . . . . . . . . . . . . . . . . . . . . 187

References . . . . . . . . . . . . . . . . . . . . . . . . . . . . . . 189

\section{Introduction}

The Minimal Model Program (in short MMP), also known as Mori's program, originated in the 80's as an attempt to generalise some of the main results of the Italian school of algebraic geometry, led by Castelnuovo at the beginning of the last century. Its aim is to construct a minimal model, i.e. a good birational representative, for any complex projective variety. Over the last two decades, the Minimal Model Program, and more in general birational geometry, has witnessed an exceptional amount of work, with three main objectives: prove some of the main outstanding conjectures of Mori's program, apply techniques from birational geometry to solve some open problems in other fields of mathematics and, finally, extend the main ideas of the program to different contexts, such as to the category of varieties defined over fields of positive characteristic, the category of Kähler vaireities or the category of foliated varieties. The goal of this note is to survey some of these achievements. It is by far not exhaustive and it does not cover many interesting aspects of the program, as it mainly focus on the author's expertise (e.g. see [33,58] for other important recent directions).

$\triangle$ Paolo Cascini

p.cascini@imperial.ac.uk

1 Department of Mathematics, Imperial College London, 180 Queen's Gate, London SW7 2AZ, UK 
We begin by a general overview of the Minimal Model Program. Although we refer to [37] for an introduction to its main techniques, we provide here a gentle introduction to some of its main goals. To this end, as in [13], we use some basic notions of graph theory, aiming to explain some of the tools and objectives in birational geometry. In particular, recall that a directed graph is a set of vertices connected by oriented edges, i.e. ordered pairs of vertices, denoted by $X \rightarrow Y$. A chain $X_{1} \rightarrow X_{2} \rightarrow \cdots$ is a sequence of distinct vertices connected by consecutive edges and a cycle $X_{1} \rightarrow X_{2} \rightarrow \cdots \rightarrow X_{n}=X_{1}$ is a finite chain starting and ending at the same vertex. A tree is a directed graph which does not contain any non-trivial cycle. Given two vertices $X$ and $Y$ of a directed graph, we say that $Y$ is below $X$ (or $X$ is above $Y$ ) if we can find a chain starting from $X$ and ending in $Y$. An end-point for a directed graph is a vertex which does not admit any other vertex below it.

We begin by considering the category of smooth projective surfaces. To this end, we construct a directed graph whose vertices are isomorphism classes of smooth projective surfaces defined over the field of complex numbers. By abuse of notation, given any smooth projective surface $X$, we also denote by $X$ the vertex representing its isomorphism class. We define the graph so that two vertices $X$ and $Y$ admit an oriented edge $X \rightarrow Y$ if and only if the surface $X$ is the blow-up of $Y$ at a closed point $y \in Y$. It turns out that the connected component containing a projective surface $X$ coincides with its birational class. Recall that two varieties are birational if they contain isomorphism dense open Zariski subsets. In particular, the connected component containing the projective plane $\mathbb{P}^{2}$ coincides with the set of complex rational surfaces. It is easy to check that this graph is a tree. Indeed, if $X, Y$ are non-isomorphic projective surfaces such that $X$ is above $Y$, i.e. there exists a non-trivial proper birational morphism $f: X \rightarrow Y$, then the second Betti number of $X$ is greater than the one of $Y$. Thus, the claim follows easily.

Note that there are always infinitely many vertices above a vertex associated to a projective surface $X$, as it is always possible to blow-up a sequence of points to obtain an arbitrary long chain above $X$. On the other hand, using the same inequality on the second Betti number as above, it is easy to check that, starting from a vertex $X$, we can find an end-point below $X$, as there does not exist any infinite chain starting from $X$. We can think of the end-point $Y$ to be a good representative of the connected class of $X$. We will see that also in higher dimension, one the main goals of the MMP is to find the end-point (or end-points) of a connected component associated to a projective variety $X$.

Projective surfaces can be divided into two large classes and the same dichotomy is expected to hold also in higher dimension. First, we assume that $X$ is a smooth projective surface which admits a global pluri-canonical form, i.e. such that

$$
h^{0}\left(X, \mathcal{O}_{X}\left(m K_{X}\right)\right)>0
$$

for some positive integer $m$, where $K_{X}$ denotes the canonical divisor of $X$. Then the subgraph obtained by considering the vertices below $X$ and the corresponding edges is finite. In addition, there exists a unique vertex which is an end-point for the connected component containing $X$. Such a vertex $Y$ is called the minimal model of $X$ and, by Castelnuovo's theorem, it is characterised by the fact that it does not admit any smooth rational curve $E$ of self-intersection -1 . Alternatively, $Y$ is the only surface in the connected component of $X$ such that $K_{Y}$ is nef, i.e. $K_{Y} \cdot C \geq 0$ for any curve $C$ in $Y$.

We now assume that $X$ is a smooth projective surface which does not admit any global pluri-canonical form, i.e. such that $h^{0}\left(X, \mathcal{O}_{X}\left(m K_{X}\right)\right)=0$ for all positive integer $m$. In this case, $X$ is uniruled, i.e. it is covered by rational curves. It is possible to show that although the graph below $X$ might be finite, there are always infinitely many end-points for the connected component of $X$. For example, if we consider again the connected component 
of rational surfaces then $\mathbb{P}^{2}$ is an end-point of such a graph, but also each Hirzebruch surface $\mathbb{F}_{n}=\mathbb{P}\left(\mathcal{O}_{\mathbb{P}^{1}} \oplus \mathcal{O}_{\mathbb{P}^{1}}(-n)\right)$, with $n \in \mathbb{N}$ and $n \neq 1$, is such.

\section{Classical Minimal Model Program}

The idea of Mori's Program is to extend the directed graph we constructed in the previous section to the set of higher dimensional varieties. It turns out that, in order to obtain results similar to the case of surfaces, it is not sufficient to consider only smooth projective varieties, nor blow-ups of closed subvarieties. We therefore now briefly describe the category of varieties which will be the vertices of our directed graph. First, we assume that these varieties are $\mathbb{Q}$-factorial, i.e. we assume that a vertex $X$ is a normal variety and any Weil divisor $S$ on $X$ is such that $m S$ is Cartier for some positive integer $m$. This allows us to take the pull-back of $S$ with respect to any morphism $W \rightarrow X$ and, in particular, define the intersection number $S \cdot C$ for any curve $C$ in $X$. Moreover, we assume that $X$ admits terminal singularities (in short $X$ is terminal), which means that for any proper birational morphism $f: Y \rightarrow X$ we have that the relative canonical divisor $K_{Y}-f^{*} K_{X}$ can be written as $\sum a_{E} E$ where the sum runs over all the exceptional prime divisors $E$ of $f$, i.e. the union $\cup E$ coincides with the locus where $f$ is not an isomorphism, and, for each such $E, a_{E}$ is a positive rational number.

Let's assume now that $X$ is a $\mathbb{Q}$-factorial terminal complex projective variety of dimension $n$ which is not minimal. This means that there exists a curve $C$ of negative canonical degree, i.e. $K_{X} \cdot C<0$. The cone theorem (cf. [37, Teorem 3.7]) and the base point free theorem (cf. [37, Teorem 3.3]) imply that, after possibly replacing $C$ by a suitable rational curve which is also of negative canonical degree, there exists a morphism $\phi: X \rightarrow Y$ with connected fibres and such that a curve $C^{\prime}$ is contracted to a point if and only if some positive rational multiple of $C^{\prime}$ is numerically equivalent to $C$, i.e. there exists $\alpha \in \mathbb{Q}_{>0}$ such that $D \cdot C^{\prime}=\alpha D \cdot C$ for any divisor $D$ in $X$. Let $E \subset X$ be the exceptional locus of $X$, i.e. the locus spanned by all such curves. We distinguish three cases:

1. If $E=X$ then $Y$ has dimension lower than $X$, and the morphism $\phi$ is called a Mori fibre space. It has the property that the relative Picard number $\rho(X / Y):=\rho(X)-\rho(Y)$ is equal to one and there exists a Zariski open subset $U \subset Y$ such that the fibre $F:=\phi^{-1}(y)$ is a terminal Fano variety for any $y \in U$, i.e. the anti-canonical divisor $-K_{F}$ of $F$ is ample.

2. If $E \subset X$ is a divisor, i.e. a proper sub-variety of dimension $n-1$, then $\phi$ is called a divisorial contraction. In this case, $Y$ is also a $\mathbb{Q}$-factorial projective variety with terminal singularities. Moreover, as in 1 , we have that the relative Picard number $\rho(X / Y)$ is equal to one.

3. Finally, if $E \subset X$ has codimension greater than one, then $\phi$ is called a flipping contraction. In this case, $Y$ is never $\mathbb{Q}$-factorial, but there exists a terminal $\mathbb{Q}$-factorial variety $X^{+}$with a morphism $\phi^{+}: X^{+} \rightarrow Y$ such that the exceptional locus of $\phi^{+}$has also codimension greater than one, there exists a curve $C^{+} \subset X^{+}$such that $K_{X^{+}} \cdot C^{+}>0$ and any curve $C^{\prime}$ in $X^{+}$is contracted by $\phi^{+}$if and only if some positive rational multiple of $C^{\prime}$ is numerically equivalent to $C^{+}$. The induced birational map $X \rightarrow X^{+}$is called a flip of $C$. The existence of a flip was proven by Mori [47] in dimension three and in full generality in $[6,26]$.

We are now ready to define our directed graph. Fix a positive integer $n$. We define the vertices of our graph to be isomorphism classes of $n$-dimensional complex projective $\mathbb{Q}$ factorial varieties with terminal singularities and given two such varieties $X$ and $X^{\prime}$, an edge 
from $X$ to $X^{\prime}$ corresponds to either a divisorial contraction $X \rightarrow X^{\prime}$ or a flip $X \rightarrow X^{\prime}$. As in the case of projective surfaces, it turns out that the graph is a tree and the connected component of a vertex $X$ coincides with the birational class of $X$. Note that, by Hironaka's theorem, any connected component contains smooth projective varieties. Moreover, since for any divisorial contraction $X \rightarrow X^{\prime}$ we have the inequality of Picard number $\rho(X)>\rho\left(X^{\prime}\right)$, it follows that there does not exist an infinite sequence of divisorial contractions. In dimension three, Shokurov proved termination of flips, which, as in the case of surfaces, implies that there does not existence an infinite chain starting from a projective variety $X$ (e.g. see [37]). In particular, for threefolds, any connected component admits at least one end-point. Up to now, it is unknown if the same property holds in higher dimension. But, existence of endpoints is know to hold true in any dimension for two large families of varieties [6]:

- A terminal projective variety $X$ is said to be of general type, if $K_{X}$ is big. Recall that a divisor $D$ on $X$ is said to be big if there exists a positive constant $C$ such that $\operatorname{dim} H^{0}\left(X, \mathcal{O}_{X}(m D)\right)>C m^{\operatorname{dim} X}$ for any sufficiently divisible positive integer $m$.

- A terminal projective variety $X$ is said to be uniruled if it is covered by rational curves, i.e. for any point $x \in X$ there exists a non trivial morphism $f: \mathbb{P}^{1} \rightarrow X$ such that $f\left(\mathbb{P}^{1}\right) \ni x$. It is possible to check that in this case, $H^{0}\left(X, \mathcal{O}_{X}\left(m K_{X}\right)\right)=0$ for any positive integer $m$. The Abundance conjecture predicts that the converse is also true.

Both these properties are birationally invariant, i.e. if $X$ is of general type (resp. uniruled) then any variety in the same connected component of $X$ is of general type (resp. uniruled). For varieties of general type, an end-point is a minimal model of general type, i.e. a projective variety with canonical divisor big and nef. For uniruled varieties, an end-point admits a Mori fibre space, as in (1). It is expected that any variety, which is not uniruled, is birational to a minimal model. Moreover, the Abundance conjecture also predicts that if $X$ is a minimal model, then there exists a morphism $\eta: X \rightarrow Z$ such that $m K_{X}=\eta^{*} H$ for some positive integer $m$ and some ample divisor $H$ on $Z$. In particular, it follows that there exists a dense Zariski open subset $U \subset Z$ such that, for any $z \in U$, the fibre $F:=\eta^{-1}(z)$ is a terminal variety with numerically trivial canonical divisor, i.e. $m K_{F} \sim 0$, for some positive integer $m$. Finally, note that varieties of general type only admit finitely many minimal models [6], i.e. their connected components only admit finitely many endpoints. On the other hand, there are varieties which are not uniruled and which admit infinitely many minimal models (e.g. see [12]).

\subsection{K-stable varieties}

Thus, at least conjecturally, the Minimal Model Program implies that, up to birational equivalence, any complex projective variety $X$ can be decomposed into varieties which belong to one of the three following families:

- Fano varieties, i.e. varieties with ample anti-canonical divisor;

- Calabi-Yau varieties, i.e. varieties with numerically trivial canonical divisor; and

- Canonically polarised varieties, i.e. varieties with ample canonical divisor.

These are usually referred to as the three building blocks in birational geometry. Note that, any complex projective manifold $X$ which belongs to one of these families admits a metric with definite Ricci curvature [3,60]. More precisely, the curvature is positive if $X$ is Fano, zero if $X$ is Calabi-Yau and negative if $X$ is canonically polarised. Furthermore, in the second and third case, we may assume that the curvature is constant, i.e. $X$ admits a Kähler-Einstein metric (in short KE metric) [60]. The existence of a KE metric on a Fano manifold is instead 
a much more difficult problem. Even in the case of surfaces, it is a well-known fact that its existence is not always guaranteed [53]. For example, the blow-up of $\mathbb{P}^{2}$ at 1 or 2 distinct points is a two-dimensional Fano manifold which does not admit a KE metric. On the other hand, the Yau-Tian-Donaldson Conjecture [23,54], which is now known to hold true (e.g. see [21]), gives a characterisation of Fano manifolds which admit a KE metric in terms of algebraic geometry. For this reason, nowadays K-stable Fano varieties play an important role both in Kähler geometry and in birational geometry (e.g. see [59] and the references therein for the definitions and a recent survey). In particular, even though a Fano variety does not admit a KE metric in general and, therefore, it is not $\mathrm{K}$-stable, we expect that it is birational to a variety which admits a Mori fibre space whose general fibre is K-stable. More, in generally, we expect:

Conjecture 2.1 Let $X$ be a smooth uniruled variety. Then $X$ is birational to a variety $Y$ which admits a morphism $\eta: Y \rightarrow Z$ such that

1. $\operatorname{dim} Z<\operatorname{dim} Y$;

2. the relative Picard number $\rho(Y / Z)$ of $\eta$ is one; and

3. the general fibre is a K-stable Fano variety.

Let us consider first the case of a rational variety $X$. Then $X$ is birational to $\mathbb{P}^{n}$ which admits a KE metric, that is the Fubini-Study metric. Thus, $X$ is birational to a $K$-stable Fano variety. On the opposite side, assume that $X$ is a birationally rigid variety. This, roughly speaking, means that $X$ is not birational to any other variety which admits a Mori fibre space and, in particular, it implies that the birational automorphism group

$$
\operatorname{Bir}(X):=\{\phi: X \rightarrow X \mid \phi \text { is a birational map }\} .
$$

is finite and coincides with the automorphism group $\operatorname{Aut}(X)$ (e.g. see [36] for a recent survey). It is reasonable to expect that if $X$ is a birationally rigid Fano variety with Picard number one then $X$ is K-stable. Consider, for example the case of a smooth hypersurface $X_{d} \subset \mathbb{P}^{n+1}$ of degree $d$. Note that $X_{d}$ is Fano if and only if $d \leq n+1$. If $d=1$ or 2 then $X_{d}$ is rational, and therefore it is not birationally rigid. On the other hand, $X_{n+1}$ is both $K$-stable and birationally rigid [61]. Note that Conjecture 2.1 toghether with the abundance conjecture and the Minimal Model Program, would imply that, up to birational equivalence, we can decompose a variety $X$ into three building blocks given by (possibly singular) varieties that admit a KE metric. Note that the existence of a KE-metric on a singular K-stable Fano variety is still an open problem.

\subsection{Boundedness and moduli spaces}

One of the main goals of birational geometry is the construction of a moduli space, whose points parametrise varieties with similar properties. This can be thought of as an approach to the problem of classifying projective varieties. For example, the module space $\mathcal{M}_{g}$ of smooth curves of genus $g \geq 2$ is one of the most intensively studied varieties in mathematics. Its construction relies on geometric invariant theory (e.g. see [32]), but it turns out that it does not extend directly to higher dimensions [57]. Instead the construction of moduli spaces of varieties of general type follows the path laid out in [38], where birational geometry plays a prominent role (see [29] and [35]). As in the case of the moduli space of curves, in order to construct a moduli space for varieties of general type, it is necessary to fix some invariants. To 
this end, given an $n$-dimensional complex projective variety $X$ of general type with terminal singularities, we define the volume of $X$ as the limit

$$
\operatorname{vol}(X):=\limsup _{m \rightarrow \infty} \frac{\operatorname{dim} H^{0}\left(X, \mathcal{O}_{X}\left(m K_{X}\right)\right)}{m^{n} / n !} .
$$

The volume of a variety of general type with terminal singularities is a birational invariant and, by the asymptotic Riemann-Roch formula (e.g. see [39]), it coincides with the top self intersection of the canonical divisor of $Y$, where $Y$ is a minimal model of $X$, i.e.

$$
\operatorname{vol}(X)=K_{Y}^{n} .
$$

Note that this is a positive rational number, which is not necessarily an integer, as $K_{Y}$ might not be Cartier, and, in particular, it does not depend on the choice of the minimal model.

The study of the volume of a projective variety plays a very important role in birational geometry. For example, we have the following boundedness result:

Theorem $2.2[25,52,56]$ For any positive integer $n$, there exists positive numbers $m_{n}$ and $\epsilon_{n}$ such that, if $X$ is a n-dimensional manifold of general type then

- $\operatorname{vol}(X) \geq \epsilon_{n}$; and

- for any positive integer $m \geq m_{n}$, the linear system $\left|m K_{X}\right|$ defines a birational map $X \rightarrow Y$.

It follows that the set of terminal varieties of general type of fixed dimension and bounded volume is birationally bounded, i.e. given a positive integer $n$ and a constant $c>0$, if

$$
\mathcal{A}_{n, c}:=\{X=n-\text { dimensional smooth variety of general type such that } \operatorname{vol}(X) \leq c\}
$$

then there exists a family $f: \mathcal{X} \rightarrow B$ proper over a variety $B$ such that for any $X \in \mathcal{A}_{n, c}$ there exists $b \in B$ such that $X$ is birational to the fibre $f^{-1}(b)$. In particular it follows that the set

$$
\{\operatorname{vol}(X) \mid X=\text { smooth projective variety of general type of dimension } n\}
$$

is discrete.

Many results of the Minimal Model Program are obtained by induction on the dimension, thanks to the Kawamata-Viehweg vanishing theorem (e.g. see [37]). For this reason, in order to apply adjunction, it is useful to replace the variety $X$ by the data of the variety $X$ together with some hypersurfaces $S_{1}, \ldots, S_{k}$ of $X$. Because of the singularities that appear in the MMP, we associate to each such hypersurface a rational coefficient. More precisely, we consider pairs $(X, \Delta)$ where $X$ is a $\mathbb{Q}$-factorial projective variety and $\Delta=\sum a_{i} S_{i}$ is a $\mathbb{Q}$-divisor with coefficients $a_{1}, \ldots, a_{k} \in(0,1] \cap \mathbb{Q}$. We say that the pair is Kawamata log terminal, in short klt, (resp. terminal, canonical, log canonical) if for any proper birational morphism $f: Y \rightarrow X$, we may write

$$
K_{Y}+\Delta_{Y}-f^{*}\left(K_{X}+\Delta\right)=\sum a_{E} E
$$

where $\Delta_{Y}$ is the strict transform of $\Delta$ in $Y$, the sum runs over all the prime exceptional divisors $E$ of $f, a_{i}<1$ (resp. $a_{i} \leq 1$ ) for all $i=1, \ldots, k$ and $a_{E}>-1$ (resp. $>0, \geq 0$, $\geq-1)$ for all $E$.

In the case of complex surfaces, if $\Delta=0$ then klt singularities coincide with singularities which, locally analytically, can be written as a quotient $\mathbb{C}^{2} / G$ where $G$ is a finite group, whilst terminal surfaces are always smooth. 
We say that the pair $(X, \Delta)$ is of log general type, if the divisor $K_{X}+\Delta$ is big. By [6], we know that klt pairs of $\log$ general type admit a minimal model, i.e. a birational map $X \rightarrow Y$, such that if $\Delta_{Y}$ is the strict transform of $\Delta$ on $Y$, then $\left(Y, \Delta_{Y}\right)$ is also klt and $K_{Y}+\Delta_{Y}$ is big and nef. By $[1,2,27,28]$, it follows that Theorem 2.2 can be generalised to klt log pairs of general type. Moreover, the set

$\{\operatorname{vol}(X) \mid X=$ klt projective variety of general type of dimension $n\}$

satisfies the Descending Chain Condition, i.e. it does not admit any infinite sequence of decreasing numbers. An important consequence of this is the existence of a constant $c_{n}$ depending only on a given positive integer $n$, such that for any $n$-dimensional manifold of general type, the group $\operatorname{Bir}(X)$ has at $\operatorname{most} c \cdot \operatorname{vol}(X)$ elements [27].

\section{Minimal Model Program for foliations}

Over the last few decades, foliation theory played a very important role in birational geometry. Miyaoka introduced the use of foliations in the Minimal Model Program to solve some of the crucial cases of the Abundance Conjecture for three-dimensional complex projective varieties [44-46]. More recently, Bogomolov and McQuillan [8] used foliations to characterise projective varieties which admit a non-trivial fibration with rationally connected fibres.

Recall that a foliation $\mathcal{F}$ of rank $r$ on a normal variety $X$ is defined by a rank $r$ coherent subsheaf $T_{\mathcal{F}} \subset T_{X}$ such that

(i) $T_{\mathcal{F}}$ is saturated, i.e. $T_{X} / T_{\mathcal{F}}$ is torsion free, and

(ii) $T_{\mathcal{F}}$ is closed under Lie bracket.

The singular locus $\operatorname{Sing} \mathcal{F}$ of $\mathcal{F}$ is the set of closed points $x \in X$ such that the sheaf $N_{\mathcal{F}}:=$ $T_{X} / T_{\mathcal{F}}$ is not locally free at $x$. Note that (i) implies that the codimension of $\operatorname{Sing} \mathcal{F}$ in $X$ is greater than one. The canonical divisor of $\mathcal{F}$ is a divisor $K_{\mathcal{F}}$ such that $\mathcal{O}_{\mathcal{X}}\left(-\mathcal{K}_{\mathcal{F}}\right) \cong \operatorname{det}\left(\mathcal{T}_{\mathcal{F}}\right)$.

By the Frobenius theorem, for any closed point $x \in X \backslash(\operatorname{Sing} X \cup \operatorname{Sing} \mathcal{F})$, there exists an analytic open neighborhood $x \in U \subset X$ such that, on $U$, the foliation $\mathcal{F}$ is induced by a morphism $\phi: U \rightarrow \mathbb{C}^{q}$ where $q=\operatorname{dim} X-r$, i.e. $\left.T_{\mathcal{F}}\right|_{U}=\operatorname{Ker}\left(T_{U} \rightarrow \phi^{*} T_{\mathbb{C}^{q}}\right)$. Viceversa, any morphism $\phi: X \rightarrow Y$ with connected fibres defines a foliation $\mathcal{F}_{\phi}$, by taking the saturation of $\operatorname{Ker}\left(T_{X} \rightarrow \phi^{*} T_{Y}\right)$ in $T_{X}$. For example, if $Y$ is a curve, then it is easy to check that the canonical divisor of $\mathcal{F}_{\phi}$ is given by

$$
K_{\mathcal{F}_{\phi}}=K_{X / Y}-\sum\left(\ell_{D}-1\right) D
$$

where $K_{X / Y}:=K_{X}-\phi^{*} K_{Y}$ is the relative canonical divisor, the sum runs over all the vertical divisors $D$ and $\ell_{D}$ denotes the multiplicity of a fibre at $D$, i.e. for any point $p \in Y$, we have $\phi^{*} p=\sum \ell_{D} D$.

For any birational map $\psi: X \rightarrow Z$, there exists an induced foliation $\mathcal{F}_{Z}$ on $Z$, defined by extending the sheaf $\left.\psi_{*} T_{\mathcal{F}}\right|_{U}$ defined on the open set $U \subset X$, on which $\psi$ is an isomorphism, to the variety $Z$. A foliation $\mathcal{F}$ on a normal variety $X$ is said to be algebraically integrable if there exists a birational map $\phi: X \rightarrow Z$ such that the induced foliation $\mathcal{F}_{Z}$ is induced by a morphism with connected fibres $Z \rightarrow Y$. It is a very important, and difficult, problem to find criteria for which a given foliation is algebraically integrable.

In many aspects, the canonical divisor of a foliation plays a similar role as the canonical divisor of a variety. It is therefore natural to ask if it is possible to divide foliations in families in a similar way as we study birational classes in the classical Minimal Model Program. To 
this end, as in the previous section, we define a directed graph associated to the category of foliated varieties.

We begin by defining the singularities which are involved in this program. To begin, we assume that the underlying complex variety $X$ is $\mathbb{Q}$-factorial. A subvariety $W \subset X$ is said to be invariant if $T_{\mathcal{F}}$ factors through the tangent space of $W$, i.e. $\left.\left.T_{\mathcal{F}}\right|_{W} \rightarrow T_{X}\right|_{W}$ factors as

$$
\left.\left.T_{\mathcal{F}}\right|_{W} \rightarrow T_{W} \rightarrow T_{X}\right|_{W}
$$

We denote $\epsilon(W)=0$ if $W$ is invariant and $\epsilon(W)=1$ otherwise. Following [41], we say that $\mathcal{F}$ is terminal (resp. canonical, klt, log canonical) if, for any proper birational morphism $f: Y \rightarrow X$, we may write

$$
K_{\mathcal{F}_{Y}}=f^{*} K_{\mathcal{F}}+\sum a_{E} E
$$

where the sum is over all the prime exceptional divisor $E$ and $a_{E}$ is a rational number such that

$$
a_{E}>0 \quad(\text { resp. } \geq 0,>-\epsilon(E), \geq \epsilon(E)) .
$$

Note that foliations do not admit a smooth resolution, as in Hironaka's theorem. For example, if $\phi: X \rightarrow Y$ is a morphism with connected fibres then the induced foliation $\mathcal{F}_{\phi}$ is singular on every singular point of any fibre of $\phi$ and it is easy to see that there does not exist a blow-up of $X$ for which the induced fibration is smooth. On the other hand, it is conjectured that if $\mathcal{F}$ is a foliation on a complex normal variety $X$ then there exists a proper birational morphism $\psi: Z \rightarrow X$ such that the induced foliation $\mathcal{F}_{Z}$ admits only canonical singularities. The existence of such a resolution is known to hold in dimension two by Seidenberg's theorem (e.g. see [10]) and in dimension three by $[11,42]$.

Exactly as in the classical Minimal Model Program, we can define divisorial contractions and flips (cf. (2) and (3) in Sect. 2 ) for foliations, by replacing the canonical divisor of a variety by the canonical divisor of a foliation. Note that running a Minimal Model Program in the category of foliated varieties preserves the fact that the foliation admits canonical singularities, Thus, for any positive integers $n$ and $r$, it makes sense to define the vertices of our directed graph to be canonical foliated varieties, i.e. pairs $(X, \mathcal{F})$ where $X$ is a $n$ dimensional complex $\mathbb{Q}$-factorial projective variety and $\mathcal{F}$ is a rank $r$ foliation on $X$ with canonical singularities. We can now define the edges of our graph induced by flips and divisorial contractions, similarly as in the case of the classical MMP. In dimension two, the Minimal Model Program for foliations was carried out successfully by the work of Brunella, McQuillan and Mendes (e.g. see [10,41,43]). Note that, in contrast with the classical MMP for surfaces, the underlying output variety might admit klt singularities. Moreover, the abundance conjecture is known to be false in this set-up [41].

In higher dimension, the cone theorem for foliations is known to hold true in two important cases: for rank one foliations in any dimension [8] and for rank two foliations in dimension three [50]. Furthermore, a version of the base point free theorem holds in dimension three by $[14,15]$. Furthermore, we have:

Theorem $3.1[14,15]$ Let $X$ be a $\mathbb{Q}$-factorial projective threefold and let $\mathcal{F}$ be a co-rank one foliation on $X$. Let $\phi: X \rightarrow Z$ be a $K_{\mathcal{F}}$-flipping contraction.

Then the flip $X \rightarrow X^{+}$, with induced morphism $\phi^{+}: X^{+} \rightarrow Z$, exists.

The idea of the proof is to construct a $\mathbb{Q}$-divisor $\Delta$ in a formal neighbourhood of the curve $C$ contracted by $\phi$, such that the pair $(X, \Delta)$ is klt and $\left(K_{X}+\Delta\right) \cdot C<0$. By Artin's approximation theorem, we may run a MMP for the pair $(X, \Delta)$ which ends with the flip $X^{+}$. 
Note that the divisor $\Delta$ is obtained by constructing suitable invariant surfaces containing $C$. Thus, although indirectly, also in the case of foliations defined over a complex variety, many of the methods rely on the Kawamata-Viehweg vanishing theorem.

As in the case of the classical Minimal Model Program, we can define the edges of our graph by flips and divisorial contractions. It is now natural to ask if, for any connected component of this graph, we may find an endpoint. Over varieties of any dimension, McQuillan [40, Main Theorem] proves the existence of a minimal model for any foliation of rank one which is not uniruled, i.e. any such $(X, \mathcal{F})$ admits a birational morphism $X \rightarrow Y$ such that $K_{\mathcal{F}_{Y}}$ is nef. Recall that a foliation is said to be uniruled if the underlying variety $X$ is covered by rational curves which are tangent to the foliation. In dimension three, termination of flips for foliations of rank two holds by [51, Theorem 0.6] and therefore the existence of minimal models is guaranteed also in this case.

Motivated by the boundedness results in the classical Minimal Model Program, it is now natural to ask if it is possible to define a moduli space for foliations using methods from birational geometry. We define a a foliated pair $(X, \mathcal{F})$ to be of general type if its canonical divisor $K_{\mathcal{F}}$ is big. Similarly to the case of varieties of general type, we define its volume bysps

$$
\operatorname{vol}(X, \mathcal{F}):=\limsup _{m \rightarrow \infty} \frac{\operatorname{dim} H^{0}\left(X, \mathcal{O}_{X}\left(m K_{\mathcal{F}}\right)\right)}{m^{n} / n !} .
$$

If $(X, \mathcal{F})$ admits a minimal model $X \rightarrow Z$ then, again by the asymptotic Riemann-Roch formula, it follows that

$$
\operatorname{vol}(X, \mathcal{F}):=\left(K_{\mathcal{F}_{Z}}\right)^{\operatorname{dim} X} .
$$

Thus, as in Theorem 2.2, we may ask if, for any positive integers $n$ and $r$, we may find positive numbers $m_{n, r}$ and $\epsilon_{n, r}$ such that, for any canonical foliated variety $(X, \mathcal{F})$ of general type of rank $r$ and dimension $n$, we have

- $\operatorname{vol}(X, \mathcal{F}) \geq \epsilon_{n, r} ;$ and

- for any positive integer $m \geq m_{n, r}$ the linear system $\left|m_{\mathcal{F}}\right|$ defines a birational map $X \rightarrow Y$.

In dimension two, a partial positive answer above was provided by $[22,24,48]$, but the question is still open in general, even after assuming that the underlying variety is a surface and the foliation is induced by a fibration. More in general, we may ask if the set of numbers defined by the volume of canonical foliated variety $(X, \mathcal{F})$ of general type of rank $r$ and dimension $n$, satisfies the Descending Chain Condition.

\section{Minimal Model Program in positive characteristic}

The study of projective varieties defined over an algebraically closed field of positive characteristic is another area on which the Minimal Model Program has experienced many exciting developments over the last few decades. Indeed, many of the results in the classical MMP generalise to this setting. In the case of surfaces, the program was carried out successfully by Bombieri and Mumford [9]. In dimension three, after the work of Keel [34] and Hacon and $\mathrm{Xu}$ [31], it is known that the MMP holds in characteristic $p \geq 5$ (e.g. see [5,7,20,30]).

One of the main differences with respect to the MMP for complex varieties is the lack of vanishing theorems (e.g. see [16] and the references therein). These are often replaced by the fact that any variety $X$ defined over a field of characteristic $p>0$, admits a Frobenius 
morphism $F: X \rightarrow X$, such that, for any Cartier divisor $D$ on $X$, we have $F^{*} D=p D$. In order to take advantage of this tool, it is crucial to choose the right category of singularities which are allowed in this program. In other words, we need to carefully choose the vertices of our directed graph. While birational morphisms are used to define singularities such as terminal, klt, etc..., the Frobenius morphism can be used to define $F$-singularities. For example, by Kunz' theorem, a variety $X$ is regular if and only if $F_{*} \mathcal{O}_{X}$ is locally free. Resolution of singularities is known for varieties of dimension $n \leq 3$ defined over an algebraically closed field of positivie characteristic but, as in the classical MMP, we cannot work only with regular varieties. To this end, strongly $F$-regular singularities play a similar role as Kawamata log terminal singularities. We now recall their definition (e.g. see [49] for more details). Let $X$ be a $\mathbb{Q}$-factorial variety defined over an algebraically closed field $k$ of characteristic $p>0$, let $D$ be an effective divisor on $X$ and let $e$ be a positive integer. Then we can define an $\mathcal{O}_{X}$-module homomorphism

$$
\operatorname{Tr}_{X}^{e}(D): F_{*}^{e}\left(\mathcal{O}_{X}\left(-\left(p^{e}-1\right) K_{X}-D\right)\right) \rightarrow \mathcal{O}_{X}
$$

which sits in the following commutative diagram of $\mathcal{O}_{X}$-modules

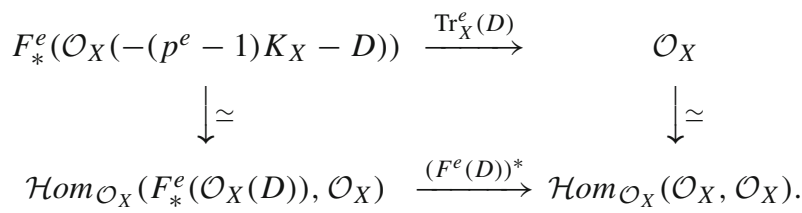

If $\Delta$ is an effective $\mathbb{Q}$-divisor on $X$, then the pair $(X, \Delta)$ is said to be strongly $F$-regular if, for every effective divisor $E$, there exists a positive integer $e$ such that $\operatorname{Tr}_{X}^{e}\left(\left\ulcorner\left(p^{e}-1\right) \Delta\right\urcorner+E\right)$ is surjective. Note that for any $\mathbb{Q}$-divisor $D=\sum a_{i} D_{i}$, we denote by $\ulcorner D\urcorner=\sum\left\ulcorner a_{i}\right\urcorner D_{i}$ the round-up of $D$. It is known that if $(X, \Delta)$ is strongly $F$-regular, then it is also klt and, as mentioned above, strongly $F$-regular singularities define a suitable category to extend many of the results of complex geometry, which holds thanks to vanishing theorems, to the category of varieties defined over $k$ (e.g. see [20]). On the other hand, klt singularities are not necessarily strongly $F$-regular (e.g. see $[18,19]$ ) and, therefore, we cannot consider the category of strongly $F$-regular pairs as a good candidate of the vertices for our directed graph. Indeed, it is easy to construct examples of strongly $F$-regular pairs such that their minimal model is not strongly $F$-regular. For this reason, many of the results of the Minimal Model Program in positive characteristic, such as the existence of flips for threefolds in characteristc $p>5$ [31], rely on comparing $F$-singularities with singularities coming from birational geometry. In small characteristic, this is still an open problem, as many pathologies are known to exist (e.g. see $[4,17,55])$.

Acknowledgements I am indebted to the organisers of the XXI conference of the Unione Matematica Italiana in Pavia for the invitation to give a talk. I would also like to thank P. Cannarsa and R. Pardini for the encouragement to publish these notes, my collaborators, from whom my research benefited enormously, and my colleagues, for giving me the opportunity to work in an excellent environment. Finally, I would like to thank the referee for many useful suggestions.

Open Access This article is licensed under a Creative Commons Attribution 4.0 International License, which permits use, sharing, adaptation, distribution and reproduction in any medium or format, as long as you give appropriate credit to the original author(s) and the source, provide a link to the Creative Commons licence, and indicate if changes were made. The images or other third party material in this article are included in the article's Creative Commons licence, unless indicated otherwise in a credit line to the material. If material is not included in the article's Creative Commons licence and your intended use is not permitted by statutory 
regulation or exceeds the permitted use, you will need to obtain permission directly from the copyright holder.

To view a copy of this licence, visit http://creativecommons.org/licenses/by/4.0/.

\section{References}

1. Alexeev, V.: Boundedness and $K^{2}$ for log surfaces. Int. J. Math. 5, 779-810 (1994)

2. Alexeev, V., Mori, S.: Bounding Singular Surfaces of General Type. Algebra, Arithmetic and Geometry with Applications (West Lafayette, IN, 2000), pp. 143-174. Springer, Berlin (2004)

3. Aubin, T.: Nonlinear Analysis on Manifolds Monge-Ampère Equations, volume 252 of Grundlehren der Mathematischen Wissenschaften [Fundamental Principles of Mathematical Sciences]. Springer, New York (1982)

4. Bernasconi, F.: Non-normal purely log terminal centres in characteristic $p \geq 3$. Eur. J. Math. 5(4), 1242-1251 (2019)

5. Birkar, C.: Existence of flips and minimal models for 3-folds in char $p$. Ann. Sci. Éc. Norm. Supér. (4) 49(1), 169-212 (2016)

6. Birkar, C., Cascini, P., Hacon, C.D., $\mathrm{M}^{\mathrm{c}}$ Kernan, J.: Existence of minimal models for varieties of log general type. J. Am. Math. Soc. 23(2), 405-468 (2010)

7. Birkar, C., Waldron, J.: Existence of Mori fibre spaces for 3-folds in char p. Adv. Math. 313, 62-101 (2017)

8. Bogomolov, F., McQuillan, M.: Rational curves on foliated varieties. In: Foliation theory in algebraic geometry, Simons Symp., pp. 21-51. Springer, Cham (2016)

9. Bombieri, E., Mumford, D.: Enriques' classification of surfaces in char. p. III. Invent. Math. 35, 197-232 (1976)

10. Brunella, M.: Birational geometry of foliations. Monografías de Matemática. Instituto de Matemática Pura e Aplicada (IMPA), Rio de Janeiro (2000)

11. Cano, F.: Reduction of the singularities of codimension one singular foliations in dimension three. Ann. Math. 160(3), 907-1011 (2004)

12. Cantat, S., Oguiso, K.: Birational automorphism groups and the movable cone theorem for Calabi-Yau manifolds of Wehler type via universal Coxeter groups. Am. J. Math. 137(4), 1013-1044 (2015)

13. Cascini, P.: Birational geometry of projective varieties and directed graphs. In: Trends in contemporary mathematics, volume 8 of Springer INdAM Ser., pp. 171-180. Springer, Cham (2014)

14. Cascini, P., Spicer, C.: MMP for co-rank one foliations on threefolds (2018). arXiv:1808.02711

15. Cascini, P., Spicer, C.: MMP for co-rank two foliations on threefolds (2020). arXiv:1912.02931

16. Cascini, P., Tanaka, H.: Smooth rational surfaces violating Kawamata-Viehweg vanishing. Eur. J. Math. 4(1), 162-176 (2018)

17. Cascini, P., Tanaka, H.: Purely log terminal threefolds with non-normal centres in characteristic two. Am. J. Math. 141(4), 941-979 (2019)

18. Cascini, P., Tanaka, H., Witaszek, J.: On log del Pezzo surfaces in large characteristic. Compos. Math. 153(4), 820-850 (2017)

19. Cascini, P., Tanaka, H., Witaszek, J.: Klt del Pezzo surfaces which are not globally $F$-split. Int. Math. Res. Not. IMRN 7, 2135-2155 (2018)

20. Cascini, P., Tanaka, H., Xu, C.: On base point freeness in positive characteristic. Ann. Sci. Ecole Norm. Sup. 48(5), 1239-1272 (2015)

21. Chen, X., Donaldson, S., Sun, S.: Kähler-Einstein metrics and stability. Int. Math. Res. Not. IMRN 8, 2119-2125 (2014)

22. Chen, Y.-A.: Boundedness of minimal partial du val resolutions of canonical surface foliations (2019). arXiv:1912.0293124

23. Donaldson, S.K.: Scalar curvature and projective embeddings. I. J. Differ. Geom. 59(3), 479-522 (2001)

24. Hacon, C., Langer, A.: On birational boundedness of foliated surfaces. Journal für die reine und angewandte Mathematik (2019) (to appear)

25. Hacon, C., $\mathbf{M}^{\mathrm{c}}$ Kernan, J.: Boundedness of pluricanonical maps of varieties of general type. Invent. Math. 166(1), 1-25 (2006)

26. Hacon, C., $\mathrm{M}^{\mathrm{c}}$ Kernan, J.: Existence of minimal models for varieties of log general type II. J. Am. Math. Soc. 23(2), 469-490 (2010)

27. Hacon, C., $\mathbf{M}^{\mathrm{c}}$ Kernan, J., Xu, C.: On the birational automorphisms of varieties of general type. Ann. Math. (2) 177(3), 1077-1111 (2013)

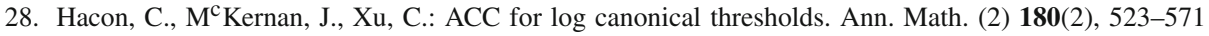
(2014) 
29. Hacon, C., $\mathbf{M}^{\mathrm{c}}$ Kernan, J., Xu C.: Boundedness of varieties of log general type. In: Algebraic geometry: Salt Lake City 2015, volume 97 of Proc. Sympos. Pure Math., pp. 309-348. Am. Math. Soc., Providence, RI (2018)

30. Hacon, C., Witaszek, J.: The minimal model program for threefolds in characteristic five (2019)

31. Hacon, C., Xu, C.: On the three dimensional minimal model program in positive characteristic. J. Am. Math. Soc. 28(3), 711-744 (2015)

32. Harris, J., Morrison, I.: Moduli of Curves Graduate Texts in Mathematics, vol. 187. Springer, New York (1998)

33. Höring, A., Peternell, T.: Minimal models for Kähler threefolds. Invent. Math. 203(1), 217-264 (2016)

34. Keel, S.: Basepoint freeness for nef and big line bundles in positive characteristic. Ann. Math. (2) 149(1), 253-286 (1999)

35. Kollár, J.: Families of varieties of general type. IHES (2017). Preprint

36. Kollár, J.: The rigidity theorem of Fano-Segre-Iskovskikh-Manin-Pukhlikov-Corti-Cheltsov-de Fernex-Ein-Mustaţă-Zhuang (2018)

37. Kollár, J., Mori, S.: Birational Geometry of Algebraic Varieties,volume 134 of Cambridge Tracts in Mathematics. Cambridge University Press, Cambridge (1998)

38. Kollár, J., Shepherd-Barron, N.: Threefolds and deformations of surface singularities. Invent. math. 91, 299-338 (1988)

39. Lazarsfeld, R.: Positivity in Algebraic Geometry. I, volume 48 of Ergebnisse der Mathematik und ihrer Grenzgebiete. Springer, Berlin (2004)

40. McQuillan, M.: Semi-stable reduction of foliations (2004). IHES Preprint

41. McQuillan, M.: Canonical models of foliations. Pure Appl. Math. Q. 4(3, part 2), 877-1012 (2008)

42. McQuillan, M., Panazzolo, D.: Almost étale resolution of foliations. J. Differ. Geom. 95(2), 279-319 (2013)

43. Mendes, L.G.: Kodaira dimension of holomorphic singular foliations. Bol. Soc. Brasil. Mat. (N.S.) 31(2), 127-143 (2000)

44. Miyaoka, Y.: Deformations of a morphism along a foliation and applications. In: Algebraic geometry, Bowdoin, 1985 (Brunswick, Maine, 1985), volume 46 of Proc. Sympos. Pure Math., pp. 245-268. Am. Math. Soc., Providence, RI (1987)

45. Miyaoka, Y.: Abundance conjecture for minimal threefolds: $v=1$ case. Comp. Math. 68, 203-220 (1988)

46. Miyaoka, Y.: On the Kodaira dimension of minimal threefolds. Math Ann. 281, 325-332 (1988)

47. Mori, S.: Flip theorem and the existence of minimal models for 3-folds. J. Am. Math. Soc. 1(1), 117-253 (1988)

48. Pereira, J.V., Svaldi, R.: Effective algebraic integration in bounded genus. Algebr. Geom. 6(4), 454-485 (2019)

49. Schwede, K.: F-adjunction. Algebra Number Theory 3(8), 907-950 (2009)

50. Spicer, C.: Higher-dimensional foliated Mori theory. Compos. Math. 156(1), 1-38 (2020)

51. Spicer, C., Svaldi, R.: Local and global applications of the minimal model program for co-rank one foliations on threefolds (2019). arXiv:1908.05037

52. Takayama, S.: Pluricanonical systems on algebraic varieties of general type. Invent. Math. 165(3), 551587 (2006)

53. Tian, G.: On Calabi's conjecture for complex surfaces with positive first Chern class. Invent. Math. 101(1), 101-172 (1990)

54. Tian, G.: Kähler-Einstein metrics with positive scalar curvature. Invent. Math. 130(1), 1-37 (1997)

55. Totaro, B.: The failure of Kodaira vanishing for Fano varieties, and terminal singularities that are not Cohen-Macaulay. J. Algebr. Geom. 28(4), 751-771 (2019)

56. Tsuji, H.: Pluricanonical systems of projective varieties of general type. II. Osaka J. Math. 44(3), 723-764 (2007)

57. Wang, X., Xu, C.: Nonexistence of asymptotic GIT compactification. Duke Math. J. 163(12), 2217-2241 (2014)

58. Wemyss, M.: Flops and clusters in the homological minimal model programme. Invent. Math. 211(2), 435-521 (2018)

59. Xu, C.: $K$-stability of Fano varieties: an algebraic geometric approach (2019). Preprint

60. Yau, S.-T.: On the Ricci curvature of a compact Kähler manifold and the complex Monge-Ampère equation. I. Comm. Pure Appl. Math. 31(3), 339-411 (1978)

61. Ziquan, Z.: Birational superrigidity and K-stability of Fano complete intersections of index one (with an appendix written jointly with C. Stibitz) (2018). arXiv:1802.08389

Publisher's Note Springer Nature remains neutral with regard to jurisdictional claims in published maps and institutional affiliations. 